\title{
Temperature dependent partition functions and equilibrium constant for HCN and HNC
}

\author{
R.J. Barber, Gregory J. Harris and Jonathan Tennyson \\ Department of Physics and Astronomy, \\ University College London, London WC1E 6BT, UK
}

\begin{abstract}
$A b$ initio vibration-rotation energy levels are summed to estimate a partition function for the total HCN system. By assigning individual levels to HCN and HNC, separate partition functions are obtained for the isomers. These are used to give a temperature dependent equilibrium constant which suggests that at temperatures typical of cool carbon stars, about $20 \%$ of the HCN system is actually HNC. Errors in the partition functions and equilibrium constant are estimated.
\end{abstract}




\section{INTRODUCTION}

The HCN and HNC linear molecules provide an important and well-studied prototype of an isomerizing system. In particular these molecules are probably the only chemically bound isomers amenable to full, accurate $a b$ initio study not only of their electronic potential energy surfaces, see for example[1-4], but also their nuclear motion states up to and including those 'delocalized' states which link the two linear minima [5-10].

The HCN system is important in its own right. It is a trace constituent of our own atmosphere and is widely seen in space. In contrast, the high abundance of the metastable HNC isomer which is frequently observed in cold, dense molecular clouds [11, 12] remains something of a mystery. Such non-LTE (local thermodynamic equilibrium) behaviour is outside the scope of the present study. Of more relevance is the presence of HCN in the atmospheres of cool carbon stars where it provides a major source of opacity [13, 14]. The atmospheres of cool stars are LTE environments and HCN is widely observed at temperatures of $2000-3000 \mathrm{~K}$. As we show below, at such temperatures there should also be a significant amount of $\mathrm{HNC}$ present in the atmospheres.

There are various methods of computing molecular partition functions. One that we have exploited successfully for water, up to temperatures of over $5000 \mathrm{~K}[15,16]$, is the explicit summation of energy levels obtained from variational nuclear motion calculations. No calculation has yet obtained all the bound rotation-vibration energy levels for a chemically bound triatomic. This means that in practise, in order to obtain convergence at higher temperatures, explicit summations over calculated energy levels must be augmented with estimates for higher-lying levels not otherwise included. This is the strategy that we employ here.

In this work we compute three separate temperature-dependent partition functions: one for the whole $\mathrm{HCN}$ system, one for $\mathrm{HCN}$ and one for HNC. Clearly, to obtain the last two partition functions it is necessary to identify individual energy levels as belonging to either $\mathrm{HCN}$ or $\mathrm{HNC}$. We then use these two partition functions to estimate the $\mathrm{HNC} \leftrightarrow$ HCN equilibrium constant as a function of temperature. Thermodynamic data for HCN, computed from its partition function, is available in JANAF [17] and fits to the JANAF partition function itself have been presented by Irwin [18, 19] over an extended temperature range. However the spectroscopic data used by JANAF and hence Irwin to generate the 
partition function is both old and very limited. Whereas there are some data on the HCN partition function, there appears to be no data available for HNC. There is only one previous estimate for the equilibrium constant, an unreliable empirically-determined one by Maki and Sams [20] which gave an energy separation between the isomers significantly lower than all previous and subsequent determinations.

As our calculations use energy levels from first principles, variational nuclear motion calculations [21, 22] based on the use of an ab initio potential energy surface [4], they themselves can be considered to be ab initio.

\section{METHOD}

The present calculations are based on the energy levels calculated by Harris et al [22] who used them and the associated wavefunctions to give a comprehensive line-list of transitions for the $\mathrm{HCN} / \mathrm{HNC}$ system. These energy levels were found by solving the bound state nuclear motion for the VQZANO+ potential of Van Mourik et al [4]. This surface was obtained by combining $a b$ initio electronic structure calculation for the isomerizing $\mathrm{HCN} / \mathrm{HNC}$ system at several levels of theory including explicit allowance for electronic relativistic effects and the adiabatic correction to the Born Oppenheimer approximation. Although not as accurate for the known energy levels as surfaces that have been empirically adjusted to spectroscopic data [23], the VQZANO+ is the best available $a b$ initio potential for the system. With this surface the $\mathrm{HNC}$ metastable isomer lies at $\Delta E_{\mathrm{HNC}}=5185.6 \mathrm{~cm}^{-1}$ above the $\mathrm{HCN}$ isomer, once allowance is made for zero point energy corrections. This prediction is in agreement with the experimental results of Pau and Hehre [24], who obtained $5180 \pm 700 \mathrm{~cm}^{-1}$, rather than $3600 \pm 400 \mathrm{~cm}^{-1}$ given by Maki and Sams [20]. Pau and Hehre's result is supported by a recent re-analysis by Wenthold [25].

The VQZANO+ potential gives a barrier to isomerization which is $16798 \mathrm{~cm}^{-1}$ above the HCN minimum [4]. Delocalized states, which cross the barrier and therefore cannot be uniquely associated with a particular isomer, occur at energies above $15000 \mathrm{~cm}^{-1}$ relative to the HCN ground state, see Bowman et al. [10] for a detailed discussion of this.

Harris et al used the exact kinetic energy operator nuclear motion program suite DVR3D [26] to obtain rotation-vibration energy levels and corresponding wavefunctions. They considered all levels of the system with rotational angular momentum, $J$, up to 60 which lay 
up to $18000 \mathrm{~cm}^{-1}$ above the ground state of $\mathrm{HCN}$, which is taken below as our zero of energy. Details of the calculation procedure used are given in ref. [22] and the levels can be obtained by either anonymous ftp from ftp.tampa.phys.ucl.ac.uk and changing to the directory /pub/astrodata/HCN, or via the web page http://www.tampa.phys.ucl.ac.uk and following the links for "Astrodata and Molecular data" then "Astrodata" then "HCN".

Comparisons with experiment $[4,21]$ suggest that the vibrational spacings agree with experiment to within a few $\mathrm{cm}^{-1}$, or $0.15 \%$, in most cases with the worst case being the $\mathrm{HNC} v_{1}$ fundamental mode where the error rises to $0.4 \%$. The calculations are particularly good at reproducing the observed rotational structure of the system [21]; the errors for the rotational levels are therefore less than the vibrational ones. These systematic errors dominate the errors in our partition functions at low temperature.

Altogether, Harris et al [22] computed over 168000 energy levels for the HCN/HNC system. At temperatures where this number of levels is sufficient to converge the summation, computation of the partition function for the total system can be achieved by a straightforward summation over these levels. However, to obtain separate partition functions for $\mathrm{HCN}$ and $\mathrm{HNC}$ is more difficult. This is because the energy levels obtained by Harris et $a l$ are not labelled as to which isomer they belong to. It is therefore necessary, at least in principle, to assign each energy level to an isomer. For 168000 levels this is a formidable task and we have therefore made some simplifying assumptions discussed below which mean that in practise we have explicitly assigned only approximately 10000 levels to an isomer. The validity of several of these assumptions can be checked by showing that they result in a negligible error in the equivalent partition function for the total system.

Both $\mathrm{HCN}$ and $\mathrm{HNC}$ are linear molecules. This means that for states with $J>0$ and $l>0$ there are two, nearly degenerate states, conventionally designated as $e$ and $f$. For $J=0$ only the $e$ state exists. Since the splitting between $e$ and $f$ states is very small we have only analysed the $e$ states and doubly weighted those levels with $l>0$.

To analyze the energy level data, all data for $e$ states with $J \leq 38$ was read into a spreadsheet. This data was then aligned so that states with the same $J$ were all in the same column and states with a common set of vibrational quantum numbers and belonging to same isomer were in the same row. As there were many state crossings in the original, purely energy-ordered, data many re-alignments were necessary. States were aligned by computing the rigid rotor quantum number, $B_{v}$, using a formula based on three neighboring 
states. In the course of sorting the data it was also possible to assign the vibrational angular momentum quantum number, $\ell$, for each state since energy levels only exist for which $\ell \leq J$. Thus for $J=0$ there are only states with $\ell=0$, new vibrational states appearing for $J=1$ have $\ell=1$ and so forth. For $J=14$ to 38,302 vibrational states were aligned, spanning energies up to $12500 \mathrm{~cm}^{-1}$. Because vibrational states with $\ell$ up to 12 were analysed, there are somewhat fewer states for lower $J$ values.

Having aligned the data, values for the rotational constants $B_{v}$ and $D_{v}$ were obtained for each vibrational series using the fourth-order rotational term value formula

$$
E_{r}=B_{v}\left[J(J+1)-\ell^{2}\right]-D_{v}\left[J(J+1)-\ell^{2}\right]^{2}
$$

HCN levels could readily be associated with those levels for which $B_{v} \leq 1.51 \mathrm{~cm}^{-1}$ and HNC with $B_{v}$ values above this value. However some HCN states with relatively high values of $D_{v}$ were found to have $B_{v}$ values somewhat above $1.51 \mathrm{~cm}^{-1}$. By analysing the constants for each case, isomer assignments were made to each of the vibrational states. These assignments, with only one exception, were found to agree with the previous analysis of Harris et al [21] who made incomplete but more extensive isomer assignments on the basis of dipole transition intensities. A third method of making possible isomer assignments, involving the sign of the permanent dipole moment [10], was not tested here.

At laboratory temperatures the energy levels considered above are sufficient to converge the partition function sums, but for higher temperatures it is necessary to consider the contribution from both higher rotational and higher vibrational states. In practise, because of the $(2 J+1)$ weighting in the partition function sum, the inclusion of higher rotational states is the more important at temperatures up to $4000 \mathrm{~K}$ (at higher temperatures, the omission of higher vibrational states becomes increasingly significant). States with $J>38$ were included by extrapolating using eq. (1) and the $B_{v}$ and $D_{v}$ constants whose determination is discussed above, with the exception that in the few cases where a negative value of $D_{v}$ was obtained $D_{v}=0$ was used instead. States up to $J=91$ were explicitly included in our final summation and the contribution from higher $J$ 's (up to 150) was approximated by assuming the contribution to $Q(T)$ from successive $J$ 's followed a geometric progression with a temperature dependent common multiple. The geometric factor, which includes the degeneracy factor, rose from 0.691 at $T=1000 \mathrm{~K}$ to 0.912 at $T=4000 \mathrm{~K}$, numbers that are consistent with the pattern for lower $J$ 's. At the temperatures considered here, the 
contribution to $Q$ from $J>150$ was found to be negligible.

Tests using an extra term, $H_{v}$, obtained using a sixth-order fit gave very similar results. The lower-order fit was preferred for reasons of numerical stability. In particular $B_{v}$ was found to always be at least $10^{5}$ greater than $D_{v}$ which is significantly bigger than the values of $J^{2}$ considered.

Below $1000 \mathrm{~K}$ the 302 vibrational states explicitly analysed above are sufficient to give $99.99 \%$ of the partition sums. However at $3000 \mathrm{~K}$ only about $87 \%$ of the partition function arises from these lower vibrational states. Harris et al [21] assigned approximately $70 \%$ of the energy levels between the 302 vibrational levels assigned here and the $18000 \mathrm{~cm}^{-1}$ cutoff to either $\mathrm{HCN}, \mathrm{HNC}$ or delocalized states on the basis of transition intensities. At higher energies, these assignments were subject to increasing uncertainty, nevertheless, Harris et al's assignments were used above the 302 energy level and the contribution to the partition function sums from the unassigned higher vibrational states was allocated to each isomer by assuming that the gaps in Harris's assignments had no bias to one or the other isotope. Analysis showed that approximately $40 \%$ of the higher levels are HCN and $60 \%$ HNC. The higher proportion of HNC levels at high energies is due to the much broader potential well about the HNC minimum which leads to a higher density of states. Tests suggest that this assumption leads a possible $5 \%$ error in the equilibrium constant $K$ at the highest temperatures considered.

\section{RESULTS}

Table I presents our calculated partition function for the whole HCN system, $Q$ (Total), the partition function for $\mathrm{HCN}, Q(\mathrm{HCN})$, that for $\mathrm{HNC}, \tilde{Q}(\mathrm{HNC})$, and the partition function for the delocalized states ie those that cannot be assigned to only one isomer, $\tilde{Q}$ (Deloc). The error estimates in Table I are derived from three possible sources of error (a) the underlying error in the ab initio data, (b) errors due to the approximate treatment of levels with $J>38$ and (c) errors due to possible misidentification of isomer levels. At low temperature, (a) is the dominant source of error. For $T \geq 2500 \mathrm{~K}$ there is a further source of error due to the omission of high-lying vibrational states from our model. This will be discussed below.

All partition functions in Table I are expressed using the $J=0$ state of $\mathrm{HCN}$ as the energy zero. Of course this is not the standard form for the HNC partition function, $Q(\mathrm{HNC})$, which 
should expressed relative to the lowest level of HNC. The two partition functions are related by the expression

$$
Q(\mathrm{HNC})=\exp \left(\frac{\Delta E_{\mathrm{HNC}}}{k T}\right) \tilde{Q}(\mathrm{HNC})
$$

where in this work $\Delta E_{\mathrm{HNC}}$ is taken to be $5185.6 \mathrm{~cm}^{-1}$. Figure 1 plots the partition functions as a function of temperature showing that $\tilde{Q}(\mathrm{HNC})$ forms a larger proportion of $Q$ (Total) as the temperature increases. Of course this is to be expected, but the effect is increased by the fact that the HNC side of the potential is much broader than the HCN side. This means that HNC supports a higher density of states meaning that actually $Q(\mathrm{HNC})$ is consistently bigger than $Q(\mathrm{HCN})$.

Figure 1 compares our partition functions with the most recent fit by Irwin [19] to the data in JANAF [17]. JANAF is entirely based on HCN data and so, presumably, Irwin's fit should correspond to $Q(\mathrm{HCN})$; in practise it lies intermediate between $Q(\mathrm{HCN})$ and $Q($ Total).

Maki et al [27] give room temperature values for the $Q(\mathrm{HCN})$ as 148.72 at $296 \mathrm{~K}$ and 149.94 at $298 \mathrm{~K}$. These values are consistent with our calculations for $Q(\mathrm{HCN})$ which are $148.53 \pm 0.54$ and $149.75 \pm 0.54$ at the same temperatures. The corresponding values for $Q(\mathrm{HNC})$ are 169.48 and 171.22 respectively, also with an error of \pm 0.54 .

Since partition functions are usually required at a range of rather precise temperatures we have fitted our data to a standard polynomial form:

$$
\log _{10} Q(T)=\sum_{i=0}^{4} a_{i}\left(\log _{10} T\right)^{i}
$$

Constants for $Q$ (Total), $Q(\mathrm{HCN})$ and $Q(\mathrm{HNC})$ are given in Table II. These constants reproduce the partition functions up to $4000 \mathrm{~K}$ to within $0.2 \%$ or better.

The partition functions for HCN and $\mathrm{HNC}$ can be used to obtain a temperature-dependent estimate of the equilibrium constant, $K$, for the simple isomerization reaction

$$
H C N \leftrightarrow H N C
$$

using the formula

$$
K=\frac{\tilde{Q}(\mathrm{HNC})}{Q(\mathrm{HCN})}=\frac{Q(\mathrm{HNC})}{Q(\mathrm{HCN})} \times \exp \left(-\frac{\Delta E_{\mathrm{HNC}}}{k T}\right) .
$$

Calculated values for $K$ are given in Table III which suggests that at temperatures above $1000 \mathrm{~K}$ there are should be appreciable quantities of HNC present in any thermalized sample. 
Indeed at the temperature of the atmosphere of cool carbon stars, up to $20 \%$ of the HCN system should be in the form of HNC. As the absorption bands $\mathrm{HNC}$ are generally stronger than those of HCN $[21,22]$, HNC should therefore provide an important source of absorption at these temperatures. Furthermore the ratio of $\mathrm{HNC} / \mathrm{HCN}$ has the potential to provide a useful temperature diagnostic.

In considering the results presented here it is important to try and assess the errors associated with what is, after all, a completely $a b$ initio theoretical treatment of the problem. In such treatments the errors are essentially all systematic which makes the giving of meaningful error bars difficult. These systematic errors essentially arise from two sources: errors in the energy levels used and errors introduced because the data set of levels used is incomplete.

The use of incomplete data necessarily leads to an underestimate of the associated partition functions and the associated error increases with $T$. Given that we allow, albeit somewhat approximately, for levels associated with $J$ values up to 150, larger errors are likely to come from the truncation of the energy levels sums at $18,000 \mathrm{~cm}^{-1}$ above the ground state. As the ground state of $\mathrm{HNC}$ is higher in energy than that of $\mathrm{HCN}$, we would expect such truncations to have a larger effect on $Q(\mathrm{HNC})$ than $Q(\mathrm{HCN})$ for a given temperature. To estimate this truncation error we modelled the higher vibrational levels by extrapolating the energy level distribution using a quadratic formula. Using this data suggests that there is no significant contribution from these levels below $2500 \mathrm{~K}$; the contributions are $0.4 \%$ at $3000 \mathrm{~K}, 1.4 \%$ at $3500 \mathrm{~K}$ and $3.2 \%$ at $4000 \mathrm{~K}$. Above this $4000 \mathrm{~K}$ this error rises rapidly and is the reason why these higher temperatures are not considered here. Our partition functions and other data have not been adjusted for this systematic error.

In addition, the assignment of levels between $\mathrm{HCN}, \mathrm{HNC}$ and delocalized states is another source of error. In the case of the first 302 levels it is possible that for those cases where $B_{v}$ is close to 1.51 some levels have been wrongly assigned. However, it is thought unlikely that any such misassignments will affect $K(T)$ by more than $1 \%$. A more significant source of error is likely to be the assumption that the gaps in Harris et al's assignments of states between the $302 \mathrm{nd}$ level and $18,000 \mathrm{~cm}^{-1}$ have no bias to one or other form of the isotope. Although not quantifiable, it is possible that at $3000 \mathrm{~K}$ this could give rise errors of up to $5 \%$.

Besides truncation errors, the other source of systematic errors in this study are associ- 
ated with the use of a particular $a b$ initio potential energy surface for the system. The error in many of the individual energy levels, particularly for low lying states, can be assessed by comparison with spectroscopic data. The potential surface generally performs well $[4,21]$ although the overestimate of $\nu_{1}$ stretching mode of HNC will lead to some underestimate of $Q(\mathrm{HNC})$. Crucial for our estimate of the equilibrium constant, $K$, and undoubtedly important also for the total partition function of the system, $Q$ (Total), is the energy separation between the ground states of the two isomers, $\Delta E_{\mathrm{HNC}}$. The separation that we have used, $5185.6 \mathrm{~cm}^{-1}$, is certainly not accurate to all the figures quoted. The figure is obtained from Harris et al's data which were obtained from calculations which made particular efforts to characterize the relative energy of the two isomers using the best possible $a b$ initio techniques [4]. Its value is consistent with most other estimates available at present but will undoubtedly be refined in the future.

We have estimated the effect on $K(T)$ of a $100 \mathrm{~cm}^{-1}$ error in the separation, $\Delta E_{\mathrm{HNC}}$. Assuming that the values for $Q(\mathrm{HCN})$ and $Q(\mathrm{HNC})$ are unaltered, changing $\Delta E_{\mathrm{HNC}}$ by 100 $\mathrm{cm}^{-1}$ alters $K$ by $5 \%$ at $2500 \mathrm{~K}$ which reduces to $4 \%$ at $4000 \mathrm{~K}$. As $T$ tends to zero, an error in $\Delta E_{\mathrm{HNC}}$ gives an increasingly large relative error in the (small) $K(T)$ value.

\section{CONCLUSIONS}

We have used rotation-vibration energy levels obtained from variational nuclear motion calculations using an $a b$ initio surface to estimate partition functions for the HCN system. From this data the calculation of the total partition function for the HCN system is fairly straightforward, however the calculation of separate partition functions for the HCN and $\mathrm{HNC}$ isomers required each level in the system to be separately labelled according to which isomer it was associated with. This task has been achieved for sufficient levels to obtain the individual partition functions and hence the temperature-dependent equilibrium constant for the HCN isomerization reaction. These calculations suggest, for example, that approaching $20 \%$ of $\mathrm{HCN}$ present in the atmosphere of cool carbon stars should be in the form of HNC, a molecule which, of course, has a completely different spectral signature. 


\section{Acknowledgments}

This project has been supported by the UK Particle Physics and Astronomy Research Council (PPARC); energy levels calculations were performed on the Miracle 24-processor Origin 2000 computer, at the HiPerSPACE Computing Centre, UCL.

[1] U. G. Jørgensen, J. Almlöf, B. Gustafsson, M. Larsson, and P. Siegbahn, J. Chem. Phys. 83, 3034 (1985).

[2] J. M. Bowman, B. Gazdy, J. A. Bentley, T. J. Lee, and C. E. Dateo, J. Chem. Phys. 99, 308 (1993).

[3] W. Jakubetz and B. Leong Lan, Chem. Phys. 217, 375 (1997).

[4] T. van Mourik G. J. Harris, O. L. Polyansky, J. Tennyson, A. G. Csaśzaŕ and P. J. Knowles, J. Chem. Phys. 115, 3706 (2001).

[5] Z. Bačić and J. C. Light, J. Chem. Phys. 86, 3065 (1987).

[6] M. Founargiotakis, S. C. Farantos and J. Tennyson, J. Chem. Phys. 88, 1598 (1988).

[7] V. Szalay, J. Chem. Phys. 92, 3633 (1990).

[8] B. Leong Lan and J. M. Bowman, J. Phys. Chem. 97, 12535 (1993).

[9] J. A Bentley, C.M. Huang and R. E. Wyatt, J. Chem. Phys. 98, 5207 (1993).

[10] J. M. Bowman, S. Irle, K. Morokuma and A. Wodtke, J. Chem. Phys. 114 (2001) 7923.

[11] T. Hirota, S. Yamamoto, M. Mikami and M. Ohishi, Astrophys. J., 503, 717 (1998).

[12] R. Bachiller, T. Forveille, P. J. Huggins and P. Cox, Astron. Astrophys., 257, 701 (1992).

[13] K. Eriksson, B. Gustafsson, U.G. Jørgensen, and $\AA$ Nordlund, Astron. Astrophys., 132, 37 (1984).

[14] R. Loidl, S. Höfner, U.G. Jørgensen, and B. Aringer, Astron. Astrophys., 342, 531 (1999),

[15] G.J. Harris, S. Viti, H.Y. Mussa and J. Tennyson, J. Chem. Phys., 109, 7197 (1998).

[16] M. Vidler and J. Tennyson, J. Chem. Phys., 113, 9766 (2000).

[17] M.W. Chase Jr, JANAF $4^{\text {th }}$ edition, J. Phys. Chem. Ref. Data, Monograph 9 (1998).

[18] A.W. Irwin, Astrophys. J. Suppl., 45, 621 (1981).

[19] A.W. Irwin, Astrophys. J. Suppl., 74, 145 (1988).

[20] A. G. Maki and R. L. Sams, J. Chem. Phys. 75, 4178 (1981). 
FIG. 1: Partition functions as a function of temperature. Solid curves: this work $Q$ (Total) highest, $Q(\mathrm{HCN})$ middle curve and $\tilde{Q}(\mathrm{HNC})$ lowest. The dashed curve is the HCN partition function given by Irwin [19].

[21] G.J. Harris, O.L. Polyansky and J. Tennyson, Spectrochimica Acta A, 58, 673 (2002).

[22] G.J. Harris, O.L. Polyansky and J. Tennyson, Astrophys. J., (in press).

[23] Q. Wu, J. Z. H. Zhang and J. M. Bowman, J. Chem. Phys. 107, 3602 (1997).

[24] C. F. Pau and W. J. Hehre, J. Phys. Chem. 86, 321 (1982).

[25] P. G. Wenthold, J. Phys. Chem. A, 104, 5612 (2000).

[26] J. Tennyson, J.R. Henderson and N.G. Fulton, Computer Phys. Comms. 86, 175 (1995).

[27] A. Maki, W. Quapp, S. Klee, G.C. Mellau and S. Albert, J. Molec. Spectrosc., 185, 360 (1997). 
TABLE I: Partition Functions of the total HCN system and its component molecules as a function of temperature. The \% error applies to both $Q($ Total $)$ and $Q(\mathrm{HCN})$.

\begin{tabular}{|c|c|c|c|c|c|}
\hline$T / \mathrm{K}$ & $Q$ (Total) & $Q(\mathrm{HCN})$ & $\tilde{Q}(\mathrm{HNC})$ & $\tilde{Q}($ Deloc $)$ & $\%$ Error \\
\hline 150 & 71.03 & 71.03 & 0 & 0 & 0.4 \\
\hline 200 & 95.49 & 95.49 & 0 & 0 & 0.4 \\
\hline 250 & 121.81 & 121.81 & 0 & 0 & 0.4 \\
\hline 300 & 150.99 & 150.99 & 0 & 0 & 0.4 \\
\hline 350 & 183.79 & 183.79 & 0 & 0 & 0.4 \\
\hline 400 & 220.87 & 220.87 & 0 & 0 & 0.3 \\
\hline 450 & 262.79 & 262.79 & 0 & 0 & 0.3 \\
\hline 500 & 310.07 & 310.07 & 0 & 0 & 0.3 \\
\hline 550 & 363.3 & 363.3 & 0 & 0 & 0.3 \\
\hline 600 & 422.9 & 422.9 & 0 & 0 & 0.3 \\
\hline 650 & 489.6 & 489.6 & 0 & 0 & 0.3 \\
\hline 700 & 564.0 & 564.0 & 0 & 0 & 0.3 \\
\hline 750 & 646.8 & 646.7 & 0 & 0 & 0.2 \\
\hline 800 & 738.6 & 738.5 & 0.1 & 0 & 0.2 \\
\hline 850 & 840.3 & 840.1 & 0.2 & 0 & 0.2 \\
\hline 900 & 952.6 & 952.2 & 0.4 & 0 & 0.2 \\
\hline 950 & 1076.5 & 1075.8 & 0.7 & 0 & 0.2 \\
\hline 1000 & 1212.8 & 1211.7 & 1.2 & 0 & 0.2 \\
\hline 1100 & 1527.2 & 1524.2 & 2.9 & 0 & 0.2 \\
\hline 1200 & 1904.5 & 1897.9 & 6.6 & 0 & 0.2 \\
\hline 1300 & 2355.1 & 2341.6 & 13.5 & 0 & 0.2 \\
\hline 1400 & 2890.6 & 2865.0 & 25.5 & 0.1 & 0.2 \\
\hline 1500 & 3523.9 & 3478.6 & 45.1 & 0.2 & 0.2 \\
\hline 1600 & 4270 & 4193 & 76 & 0.5 & 0.2 \\
\hline 1700 & 5145 & 5021 & 122 & 1 & 0.3 \\
\hline 1800 & 6166 & 5975 & 189 & 2 & 0.3 \\
\hline 1900 & 7355 & 7067 & 284 & 4 & 0.3 \\
\hline 2000 & 8731 & 8311 & 414 & 7 & 0.3 \\
\hline 2100 & 10319 & 9720 & 12588 & 12 & 0.3 \\
\hline 2200 & 12143 & 11308 & 815 & 20 & 0.4 \\
\hline 2300 & 14228 & 13089 & 1108 & 31 & 0.4 \\
\hline 2400 & 16600 & 15077 & 1477 & 47 & 0.5 \\
\hline
\end{tabular}


TABLE II: Fitted constants for fourth-order fit to $Q(\mathrm{X})$, see eq (3).

\begin{tabular}{lrrrrr} 
X & $a_{0}$ & $a_{1}$ & $a_{2}$ & $a_{3}$ & $a_{4}$ \\
Total & -46.411039 & 65.619568 & -33.437298 & 7.4856164 & -0.59928697 \\
HCN & -104.97196 & 146.91809 & -75.853937 & 17.337515 & -1.4556821 \\
HNC & -55.717312 & 79.913945 & -41.641528 & 9.5704500 & -0.79716903 \\
\hline
\end{tabular}


TABLE III: Equilibrium constant, $K$, for $\mathrm{HCN} \leftrightarrow \mathrm{HNC}$ as a function of temperature. The percentage of $\mathrm{HNC}$ for a thermalized sample is also given. (Numbers in parenthesis denote powers of ten).

\begin{tabular}{|c|c|c|}
\hline$T / \mathrm{K} K(T)$ & $\%$ Error & $\% \mathrm{HNC}$ \\
\hline $3001(-11)$ & 0.8 & $2(-9)$ \\
\hline $4001(-8)$ & 0.6 & $1(-6)$ \\
\hline $5004(-7)$ & 0.6 & $4(-5)$ \\
\hline $6006(-6)$ & 0.6 & $6(-4)$ \\
\hline $7004(-5)$ & 0.6 & $4(-3)$ \\
\hline $800 \quad 0.0001$ & 0.4 & 0.014 \\
\hline $900 \quad 0.0004$ & 0.4 & 0.040 \\
\hline $1000 \quad 0.0010$ & 0.4 & 0.095 \\
\hline 11000.0019 & 0.4 & 0.193 \\
\hline 12000.0035 & 0.4 & 0.347 \\
\hline 13000.0058 & 0.4 & 0.573 \\
\hline 14000.0089 & 0.4 & 0.881 \\
\hline 15000.0130 & 0.4 & 1.28 \\
\hline $1600 \quad 0.0181$ & 0.4 & 1.78 \\
\hline $1700 \quad 0.0243$ & 0.6 & 2.38 \\
\hline $1800 \quad 0.0317$ & 0.6 & 3.07 \\
\hline 19000.0402 & 0.6 & 3.86 \\
\hline $2000 \quad 0.0498$ & 0.6 & 4.74 \\
\hline $2100 \quad 0.0605$ & 0.6 & 5.69 \\
\hline $2200 \quad 0.0721$ & 0.8 & 6.71 \\
\hline 23000.0846 & 0.8 & 7.79 \\
\hline 24000.0980 & 1.0 & 8.90 \\
\hline $2500 \quad 0.1119$ & 1.0 & 10.0 \\
\hline $2600 \quad 0.1265$ & 1.2 & 11.2 \\
\hline $2700 \quad 0.1415$ & 1.2 & 12.3 \\
\hline $2800 \quad 0.1569$ & 1.4 & 13.5 \\
\hline 29000.1725 & 1.6 & 14.6 \\
\hline $\begin{array}{lll}3000 & 0.1882 & 14\end{array}$ & $4^{1.6}$ & 15.7 \\
\hline $3100 \quad 0.2039$ & 1.8 & 16.8 \\
\hline $3200 \quad 0.2196$ & 2.0 & 17.8 \\
\hline $3300 \quad 0.2353$ & 2.0 & 18.8 \\
\hline
\end{tabular}

